\title{
artigo
}

Furtado, M.S.; Bridi, A.C.; Louro, T.Q.; Marques, J.M.; Castro, D.F.; Lyra da Silva, R.C.

Parametrização de alarmes clínicos de monitores multiparâmetros em uma unidade de cuidados crítico

\section{Parametrização de alarmes clínicos de monitores multiparâmetros em uma unidade de cuidados crítico}

\author{
Parameterization of clinical alarms of multiparameter monitors in a critical care unit \\ Parameterización de alarmas clínicas de monitores multiparámetros en una unidad de atención crítica
}

\begin{abstract}
RESUMO
Objetivos: Identificar e classificar os alarmes sonoros de variáveis hemodinâmicas disparadas pelo monitor multiparâmetros, antes e após a intervenção de parametrização dos valores limítrofes dos alarmes. Método: Abordagem quantitativa, delineamento quase experimental, estudo de avaliação antes e depois, técnica de coleta de dados observação não participante em uma unidade de terapia intensiva, por $60 \mathrm{~h}$. Os participantes do estudo foram os profissionais de enfermagem. Produção de dados dividida em dois momentos: $1^{\circ}$ momento de caracterização dos pacientes e $2^{\circ}$ momento de descrição dos alarmes (fase pré e pós parametrização). Análise dos dados com cálculo da razão de chance (OR). Resultados: Durante as fases pré e pós, 87 pacientes foram contabilizados e 42 (48\%) encontravam-se com no mínimo um alarme desligado. Na fase pré, registraram-se 513 alarmes, 428 (83,4\%) inconsistentes, 497 (124 alarmes fatigados e 373 perdidos) não atendidos pela equipe de enfermagem. Na fase pós, registraram-se 438 alarmes, 330 (75.3\%) inconsistentes, 423 (90 alarmes fatigados e 333 perdidos) não atendidos. A OR para alarmes fatigados e alarmes consistentes é de 1.2 para o primeiro evento e 0.5 para o segundo. Conclusões: Primeiro passo ao mapeamento da fadiga de alarmes, assim como da usabilidade de equipamentos. Mapeamento que subsidiará posterior capacitação da equipe, adoção de protocolo e rotina referentes à parametrização e usabilidade, que irão concorrer no correto raciocínio clínico na tomada de decisão dos profissionais, favorecendo a vigilância do paciente e consequentemente sua segurança.
\end{abstract}

DESCRITORES: Alarmes Clínicos. Cuidados Críticos. Monitoramento. Segurança do Paciente. Enfermagem.

\section{ABSTRACT}

Objectives: To identify and classify the audible alarms of hemodynamic variables triggered by the multiparameter monitor, before and after the intervention of parameterization of the limit values of the alarms. Method: Quantitative approach, quasi-experimental design, evaluation study before and after, data collection technique, non-participant observation in an intensive care unit, for $60 \mathrm{~h}$. The study participants were nursing professionals. Data production was divided into two moments: 1st moment of patient characterization and 2nd moment of alarm description (pre and post parameterization phase). Data analysis with calculation of the odds ratio (OR). Results: During the pre and post phases, 87 patients were counted and $42(48 \%)$ had at least one alarm turned off. In the pre-phase, 513 alarms were registered, 428 (83.4\%) were inconsistent, 497 (124 fatigued alarms and 373 missed) were not attended by the nursing staff. In the post phase, 438 alarms were recorded, 330 (75.3\%) were inconsistent, 423 (90 fatigued alarms and 333 missed) were not answered. The OR for fatigued alarms and consistent alarms is 1.2 for the first event and 0.5 for the second. Conclusions: First step to map alarm fatigue, as well as equipment usability. Mapping that will subsidize the team's subsequent training, adoption of a protocol and routine regarding parameterization and usability, which will help in the correct clinical reasoning in the professionals' decision making, and will favor to the patient's surveillance and consequently their safety.

DESCRIPTORS: Clinical Alarms. Critical Care. Monitoring. Patient safety. Nursing.

\section{RESUMEN}

Objetivos: identificar y clasificar las alarmas audibles de las variables hemodinámicas activadas por el monitor multiparamétrico, antes y después de la intervención de parametrización de los valores límite de las alarmas. Método: enfoque cuantitativo, diseño cuasiexperimental, estudio de evaluación antes y después, técnica de recolección de datos, observación no participante en una unidad de cuidados intensivos, durante $60 \mathrm{~h}$. Los participantes del estudio eran profesionales de enfermería. Producción de datos dividida en dos momentos: primer momento de caracterización del paciente y segundo momento de descripción de la alarma (fase de parametrización previa y posterior). Análisis de datos con cálculo de odds ratio (OR). Resultados: Durante las fases pre y post, se contaron 87 pacientes y $42(48 \%)$ tuvieron al menos una alarma apagada. En la fase previa, se registraron 513 alarmas, 428 (83.4\%) inconsistentes, 497 (124 alarmas fatigadas y 373 perdidas) no atendidas por el personal de enfermería. En la fase posterior, se registraron 438 alarmas, 330 (75.3\%) fueron inconsistentes, 423 (90 alarmas fatigadas y 333 perdidas) no fueron respondidas. El OR para alarmas fatigadas y alarmas consistentes es 1.2 para el primer evento y 0.5 para el segundo. Conclusiones: primer paso 
para mapear la fatiga de la alarma, así como la usabilidad del equipo. Mapeo que subsidiará la capacitación posterior del equipo, la adopción de un protocolo y una rutina con respecto a la parametrización y la usabilidad, que ayudará en el razonamiento clínico correcto en la toma de decisiones de los profesionales, favoreciendo la vigilancia del paciente y, en consecuencia, su seguridad.

DESCRIPTORES: Alarmas Clínicas. Cuidado crítico. Monitoreo. Seguridad del paciente. Enfermería.

RECEBIDO EM: 09/07/2020 APROVADO EM: 14/07/2020

\section{Monique de Sousa Furtado}

Mestrado em Enfermagem pela Universidade Federal do Estado do Rio de Janeiro-Unirio. Enfermeira Intensivista no Hospital Universitário Clementino Fraga Filho da Universidade Federal do Rio de Janeiro-HUCFF-UFRJ.

ORCID: 0000-0003-1593-8766.

\section{Adriana Carla Bridi}

Doutorado em Enfermagem e Biociências Universidade Federal do Estado do Rio de Janeiro-Unirio. Enfermeira Intensivista no Hospital Universitário Pedro Ernesto-Universidade do Estado do Rio de Janeiro-Hupe-Uerj.

ORCID: 0000-0003-2018-4604

\section{Thiago Quinellato Louro}

Enfermeiro. Pós-Doutor em Enfermagem - Universidade Federal do Estado do Rio de Janeiro. Professor Adjunto, Universidade Federal Fluminense. Rio das Ostras, RJ, Brasil.

ORCID: 0000-0001-8371-628X

\section{Juliana Mendes Marques}

Mestrado em Enfermagem pela Universidade Federal do Estado do Rio de Janeiro-Unirio. Professora do curso de medicina da Universidade Estácio de Sá. Angra dos Reis-Rio de Janeiro.

ORCID: 0000-0002-6730-9649

\section{Dayane Ferreira de Castro}

Mestranda em Enfermagem pela Universidade Federal do Estado do Rio de Janeiro-Unirio. Enfermeira da Emergência Pediátrica do Hospital Estadual Adão Pereira Nunes.

ORCID: 0000-0001-9437-3673

\section{Roberto Carlos Lyra da Silva}

Coordenador do Programa de Pós Graduação Doutorado em Enfermagem e Biociências Universidade Federal do Estado do Rio de Janeiro-Unirio. Pesquisador líder do Laboratório de Avaliação Econômica e de Tecnologias em Saúde - LAETS/CNPq e Coordenador do Laboratório de Simulação e Avaliação de Usabilidade e Fator Humano da UNIRIO.

ORCID: 0000-0001-9416-9525

\section{INTRODUÇÃO}

A Unidade de Terapia Intensiva (UTI) é o espaço especializado no atendimento de pacientes que apresentam instabilidade de um ou mais sistemas orgânicos ${ }^{(1-2)}$. O doente grave, devido à sua vulnerabilidade orgânica, é dependente dos Equipamentos Médicos Assistenciais (EMAs) para suporte avançado de vida, diagnóstico, monitorização e tratamento. Contudo, estas tecnologias podem ser deletérias para a segurança do paciente, caso o usuário não esteja familiarizado com suas funcionalidades ${ }^{(3)}$.
O Emergency Care Research Institute (ECRI) divulga anualmente um relatório com os perigos das tecnologias em saúde ("Top 10 Health Technology Hazards"), citando desde 2007, os sistemas alarmes dos equipamentos como um desses perigos $^{(4)}$.

Neste cenário, pesquisadores descreveram a fadiga de alarmes. Este fenômeno se refere ao grande número de alarmes que encobre os clinicamente significativos, possibilitando que alarmes de relevância clínica sejam desabilitados, silenciados ou ignorados pela equipe. $\mathrm{O}$ número excessivo de alarmes acarreta sobrecarga sensorial e dessensibilização da equipe, reduzindo a confiança no sentido de urgência dos alarmes e levando a falta de resposta aos alarmes. Alarmes de relevância clínica quando subestimados podem resultar em graves consequências nas condições clínicas do paciente ${ }^{(5-7)}$. Essa dessensibilização da equipe de saúde em relação aos alarmes se deve, entre outros fatores, a falta de parametrização (ajuste de valores de parâmetros fisiológicos máximos e mínimos para alerta) desses alarmes, estando ainda desabilitados, com baixo volume, predispondo a desfechos indesejados aos pacientes, por situações não sinalizadas ${ }^{(7)}$. 
A Food and Drug Administration (FDA) e a Manufacturer and User Facility Device Experience (MAUDE) receberam 566 relatos de mortes de pacientes relacionadas aos alarmes de monitorização em hospitais nos Estados Unidos entre 2005 a 2008 , demonstrando que o uso incorreto dos sistemas de alarmes tem impacto direto na segurança do paciente ${ }^{(7)}$.

Em um estudo coreano, verificou-se que os enfermeiros individualizavam os alarmes em somente $9(18,8 \%)$ dos 48 pacientes assistidos em sua unidade, pois consideravam difícil a configuração correta dos monitores ${ }^{(8)}$.

No Brasil, estudo realizado em unidade coronariana, identificou $64 \%$ de alarmes não atendidos pela equipe do serviço diurno e $63 \%$ sem resposta no serviço noturno. Os resultados demonstram que a parametrização dos alarmes dos monitores no campo de pesquisa foi inadequada e que tal fato está intimamente relacionado à fadiga de alarmes ${ }^{(9)}$.

Outro estudo brasileiro realizado em um hospital público identificou que o monitor multiparâmetros foi o aparelho que mais alarmou, totalizando 68 vezes $(66,09 \%)$, seguido pela bomba infusora 25 $(24,27 \%)$ e o ventilador mecânico, com 8 $(7,76 \%)$ alarmes ${ }^{(10)}$.

Caso a individualização dos alarmes não aconteça, alarmes frequentes e desnecessários ocorrerão continuamente por motivos não passíveis de ação, o que inevitavelmente levará ao ruído, à distração e ao aumento do potencial para a fadiga de alarmes naquele ambiente ${ }^{(11)}$.

A parametrização pode ser considerada uma excelente estratégia para reduzir o número de alarmes dentro das UTIs e assim, corroborar para uma melhor vigilância por parte dos profissionais aos pacientes graves nesses ambientes e, consequentemente, garantir sua segurança ${ }^{(7)}$. Portanto, os objetivos deste estudo são identificar e classificar os alarmes sonoros de variáveis hemodinâmicas disparadas pelo monitor multiparâmetros, antes e após a intervenção de parametrização dos valores limítrofes dos alarmes.
No Brasil, estudo realizado em unidade

coronariana, identificou $64 \%$

de alarmes não

atendidos pela equipe do serviço

diurno e $63 \%$

sem resposta no

serviço noturno.

Os resultados

demonstram que

a parametrização

dos alarmes dos

monitores no

campo de pesquisa

foi inadequada $e$

que tal fato está

intimamente

relacionado à fadiga

de alarmes

\section{MÉTOdOS}

A pesquisa teve abordagem quantitativa $\mathrm{e}$ delineamento quase experimental, caracterizando um estudo de avaliação antes e depois. A técnica de coleta de dados foi observação não participante (exceto nos casos em que havia risco para o paciente). A produção de dados foi dividida em dois momentos: $1^{\circ}$ momento o de caracterização dos pacientes e o $2^{\circ}$ momento de descrição dos alarmes (fase pré e pós parametrização). As variáveis fisiológicas monitoradas pelos monitores multiparâmetros para fins de observação dos alarmes disparados na unidade foram: frequência cardíaca (FC), frequência respiratória (FR), pressão arterial não invasiva (PNI) e invasiva (PAI), considerando suas medidas de sistólica, diastólica e média.

O cenário de estudo foram 6 leitos que permitiam total visibilidade dos pacientes e audibilidade dos alarmes de uma UTI adulto, hospital da rede privada, localizada na cidade do Rio de Janeiro. O período foi de $60 \mathrm{~h}$ de observação, sendo cada fase com duração de $30 \mathrm{~h}$. A coleta ocorreu em 15 dias não consecutivos, entre segunda $\mathrm{e}$ sexta-feira, numa média de $4 \mathrm{~h}$ de observação diária no turno da tarde. A pesquisa foi aprovada pelo Comitê de Ética Pesquisa com CAAE: 59557316.9.0000.5285.

Os participantes do estudo foram os profissionais de enfermagem (enfermeiros e técnicos de enfermagem), que foram observados quanto ao atendimento aos alarmes disparados pelos monitores multiparâmetros.

Cabe destacar que alarmes inconsistentes, falsos, ou ainda irrelevantes, são aqueles que, quando disparados, representam mau funcionamento do EMA ou não se remetem a nenhuma alteração fisiológica importante ${ }^{(9)}$. O número de alarmes que soaram no período de observação foi criteriosamente contabilizado manualmente através de um cronômetro pela pesquisadora em instrumento próprio. Para que um alarme fosse considerado atendido o profissional deveria ter se dirigido a beira do leito, ainda que não tomasse qualquer conduta demandada pelo alarme sonoro.

O tempo máximo de espera cronometrado para que o profissional respondesse 
ao alarme sonoro foi de até 04 minutos. Alarmes sonoros disparados que não tiveram resposta nesse intervalo de tempo foram classificados como alarmes fatigados (sem resposta). Alarmes sonoros disparados, que se silenciaram automaticamente sem que tivessem obtido alguma resposta, também dentro desse intervalo de tempo, foram classificados como alarmes perdidos. A delimitação desse tempo teve como referência o quanto dura a fase elétrica do coração, de acordo com o conceito fase-dependente na parada cardiorrespiratória (PCR), onde é possível identificar 03 diferentes fases em uma PCR: a elétrica, que se estende do minuto zero ao quarto minuto pós-PCR; a circulatória, que vai de aproximadamente do quarto minuto a aproximadamente ao décimo minuto após a PCR, e a fase metabólica, que se instala após o décimo minuto pós-PCR ${ }^{(12)}$.

$\mathrm{Na}$ fase pré-parametrização a pesquisadora acionou os alarmes que estavam desligados sem a individualização de seus valores limítrofes de acordo com a condição clínica dos pacientes, isto é, deixando os valores limítrofes de fábrica do equipamento.
A parametrização dos alarmes sonoros das variáveis fisiológicas monitoradas pelos monitores dos leitos elencados foi de acordo com a condição clínica de cada doente, avaliada pela pesquisadora pelo registro das últimas $24 \mathrm{~h}$ de evolução dos pacientes. A individualização foi necessária, pois, embora os pacientes tenham sido separados nas categorias clínicas, existiram dentro de uma mesma categoria, indivíduos com respostas orgânicas diferenciadas a mesma proposta terapêutica.

Estabeleceu-se um acréscimo de 10\% de margem de tolerância aos valores mínimos e máximos definidos para a intervenção, considerando os sinais vitais das últimas $24 \mathrm{hs}$ de internação registrados no balanço hídrico, mesmo que o paciente estivesse em vigência de suporte, objetivando atingir o alvo terapêutico, conforme as recomendações do Summit Clinical Alar$\mathrm{ms}{ }^{(13)}$. Para a análise quantitativa dos dados, foram utilizados os recursos e funções do programa Excel'. A estatística foi utilizada para descrever e analisar as variáveis de interesse do estudo, a saber: o número de alarmes disparados pelo monitor mul- tiparâmetros e a consistência dos alarmes disparados. Foi calculado a OR ("odds ratio" ou Razão de possibilidades) da ocorrência de um alarme fatigar antes e depois da parametrização e de ter um alarme consistente antes e após a parametrização.

\section{RESULTADOS}

No primeiro momento, foram anotadas informações, por meio de um diário de campo sobre a caracterização dos pacientes internados na UTI e o Nursing Activities Score (NAS), além do registro de situações que poderiam ser relevantes e complementares ao estudo.

Sobre o NAS, instrumento para medir a carga de trabalho de enfermagem, a média foi 78 pontos. Cada ponto do NAS corresponde a 14,4 minutos, logo, a média equivale a 18.72 horas de cuidados de enfermagem por paciente em um turno de $24 \mathrm{~h}$ de trabalho.

No quadro 1, figura 1 e tabela 1 estão expostos os seguintes dados dos dois momentos da metodologia e a comparação entre eles.

\begin{tabular}{|c|c|c|c|c|}
\hline \multicolumn{5}{|l|}{ PRIMEIRO MOMENTO } \\
\hline & \multicolumn{2}{|c|}{ PRÉ-PARAMETRIZAÇÃO } & \multicolumn{2}{|c|}{ PÓS-PARAMETRIZAÇÃo } \\
\hline Número absoluto de pacientes & \multicolumn{2}{|c|}{47} & \multicolumn{2}{|c|}{40} \\
\hline Média de idade dos pacientes (anos) & \multicolumn{4}{|c|}{78 anos } \\
\hline Média de tempo de internação(dias) & \multicolumn{4}{|c|}{19 dias } \\
\hline Taxa de Mortalidade (\%) & \multicolumn{4}{|c|}{$23,75 \%$} \\
\hline Pacientes com ao menos um alarme sonoro desligado (\%) & \multicolumn{4}{|c|}{$48 \%$} \\
\hline $\begin{array}{l}\text { Pacientes com aminas vasoativas com ao menos uma variável PAI/PNI com } \\
\text { alarme sonoro desligado (\%) }\end{array}$ & \multicolumn{4}{|c|}{$44 \%$} \\
\hline Pacientes classificados na categoria clínica de sepse (100\%) & \multicolumn{2}{|c|}{$68 \%$} & \multicolumn{2}{|c|}{$62,50 \%$} \\
\hline \multirow{2}{*}{$\begin{array}{l}\text { Distribuição das variáveis fisiológicas monitoradas que estavam com alarmes } \\
\text { desligados }(100 \%)\end{array}$} & FR & PAI/PNI & FR e PAl & FR e PNI \\
\hline & $23,8 \%$ & $57,2 \%$ & $4,8 \%$ & $14,2 \%$ \\
\hline \multicolumn{5}{|l|}{ SEGUNDO MOMENTO } \\
\hline \multicolumn{5}{|c|}{ FASE PRÉ-PARAMETRIZAÇÃO } \\
\hline \multirow{4}{*}{ Alarmes Disparados (100\%) (513) } & \multicolumn{2}{|c|}{ Atendidos } & \multicolumn{2}{|c|}{ Não Atendidos } \\
\hline & \multicolumn{2}{|c|}{$3,1 \%(16)$} & \multicolumn{2}{|c|}{$96,9 \%(497)$} \\
\hline & \multicolumn{2}{|c|}{ Consistentes } & \multicolumn{2}{|c|}{ Inconsistentes } \\
\hline & \multicolumn{2}{|c|}{$16,6 \%$ (85) } & \multicolumn{2}{|c|}{$83,4 \%(428)$} \\
\hline
\end{tabular}




\section{artigo}

Furtado, M.S.; Bridi, A.C.; Louro, T.Q.; Marques, J.M.; Castro, D.F.; Lyra da Silva, R.C.

Parametrização de alarmes clínicos de monitores multiparâmetros em uma unidade de cuidados crítico

\begin{tabular}{|c|c|c|}
\hline \multirow{4}{*}{ Alarmes Disparados Não Atendidos (100\%) (497) } & Alarmes Fatigados & Alarmes Perdidos \\
\hline & $25 \%(124)$ & $75 \%(373)$ \\
\hline & Consistentes & Inconsistentes \\
\hline & $15,9 \%(79)$ & $84,1 \%(418)$ \\
\hline \multicolumn{3}{|c|}{ FASE PÓS-PARAMETRIZAÇÃO } \\
\hline \multirow{4}{*}{ Alarmes Disparados (100\%) (438) } & Atendidos & Não Atendidos \\
\hline & $3,4 \%(15)$ & $96,6 \%(423)$ \\
\hline & Consistentes & Inconsistentes \\
\hline & $24,7 \%(108)$ & $75,3 \%(330)$ \\
\hline \multirow{4}{*}{ Alarmes Disparados Não Atendidos (100\%) (423) } & Alarmes Fatigados & Alarmes Perdidos \\
\hline & $21 \%(90)$ & $79 \%(333)$ \\
\hline & Consistentes & Inconsistentes \\
\hline & $23,4 \%(99)$ & $76,6 \%(324)$ \\
\hline
\end{tabular}

Figura 1: Distribuição dos alarmes sonoros disparados por variável fisiológica monitorada de acordo com sua consistência (fases pré e pós-parametrização).

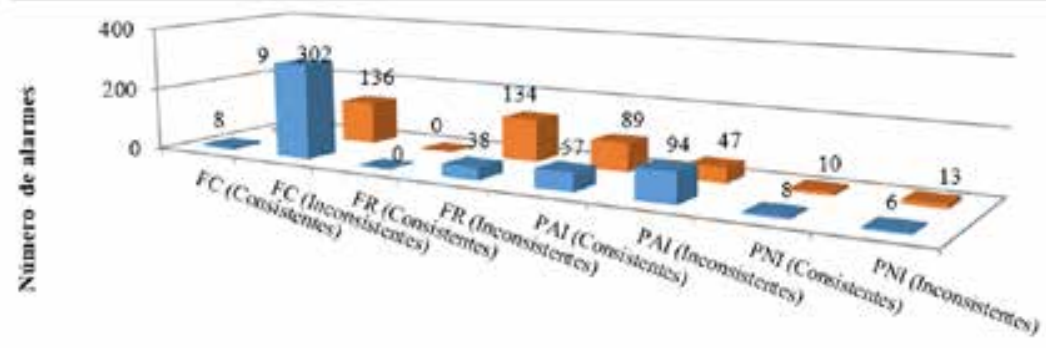

= Pré-Parametrizaçào = Pós-Parametrizaçlo

Legenda: Frequência Cardíaca (FC), Frequência Respiratória (FR), Pressão Arterial Invasiva (PAl) e Pressão Arterial Não Invasiva (PNI).

Tabela 1: Comparação do número de alarmes sonoros disparados das variáveis fisiológicas observadas nas fases pré e pós-intervenção- Unidade de Terapia Intensiva (UTI) - Rio de Janeiro, 2017.

\begin{tabular}{lcccccc} 
Variável & \multicolumn{2}{c}{$\begin{array}{c}\text { Número de alarmes } \\
\text { (pré-parametrização) }\end{array}$} & \multicolumn{2}{c}{$\begin{array}{c}\text { Número de alarmes } \\
\text { (pós-parametrização) }\end{array}$} & \multicolumn{2}{c}{$\begin{array}{c}\text { Relação de acréscimo/ } \\
\text { fecréscimo dos valores }\end{array}$} \\
Fisiológica & Quantidade & $\%$ & Quantidade & $\%$ & Quantidade & $\%$ \\
FC & 310 & $60,4 \%$ & 145 & $33,1 \%$ & Decréscimo & $53,2 \%$ \\
FR & 38 & $7,4 \%$ & 134 & $30,6 \%$ & Acréscimo & $252,6 \%$ \\
PAI & 151 & $29,4 \%$ & 136 & $31,1 \%$ & Decréscimo & $9,9 \%$ \\
PNI & 14 & $2,7 \%$ & 23 & $5,3 \%$ & Decréscimo & $64,3 \%$ \\
TOTAL & 513 & $100 \%$ & 438 & $100 \%$ & Decréscimo & $14,62 \%$ \\
Legenda: FC (frequência cardíaca); FR (frequência respiratória); PAl (pressão arterial invasiva); PNI (pressão arterial não invasiva).
\end{tabular}

Uma vez calculado a OR para a ocorrência de alarmes fatigados e alarmes con-

sistentes, obteve-se o valor de 1.2 para o primeiro evento e de 0.5 para o segundo.

\section{DISCUSSÃO}

A parametrização segura deve considerar o perfil dos pacientes e suas condiçôes clínicas, a fim de definir corretamente os limites de alarmes e assim, não contribuir para a existência da fadiga de alarmes no local ${ }^{(11)}$.

A observação criteriosa dos valores de pressão arterial, objetivando titulação de aminas vasoativas é ponto imperativo para o alcance do alvo terapêutico neste tipo de paciente. Portanto, é extremamente preocupante encontrar estas variáveis fisiológicas com seus alarmes sonoros desligados ou fora dos parâmetros de segurança.

O percentual de alarmes sonoros disparados pelos monitores considerados inconsistentes nesta pesquisa (83\%) se assemelha ao encontrado em um estudo australiano que constatou $99 \%$ de alarmes inconsistentes e em outro ocorrido em um hospital de ortopedia brasileiro cujo resultado foi de $91,1 \%$ alarmes inconsistentes ${ }^{(14-15)}$. Em um estudo coreano, os membros da equipe foram repetidamente expostos a uma média de 771 alarmes/pacientes/dia e após a experiência relataram diminuição na concentração, sentindo-se distraídos, predispondo-os a erros ${ }^{(8)}$.

O elevado percentual de alarmes inconsistentes na UTI estudada, está vinculado ao fenômeno da fadiga de alarmes, visto que o número de alarmes considerados 
fatigados foram $25 \%$ do total disparado. Profissionais que trabalham em ambientes com exposição à elevados níveis de ruídos, incluindo os oriundos dos equipamentos, podem desenvolver desordem em seu estado físico, mental e psicológico, e, por conseguintes alterações na comunicação, baixo desempenho, fadiga, estresse, doenças e até acidentes de trabalho ${ }^{(16)}$.

$\mathrm{Na}$ fase pós-intervenção o número de alarmes sonoros disparados registrados pela pesquisadora diminuiu em relação à primeira fase (antes da parametrização) numa proporção de $14.6 \%$. Nota-se que houve uma redução de quase $10 \%$ no percentual de alarmes inconsistentes entre as duas fases de observação; na primeira fase do total de alarmes registrados pela pesquisadora (428) 83\% foram inconsistentes e na segunda fase (330) 75.3\%.

Este resultado se assemelha, guardadas as devidas proporções, com 2 pesquisas que individualizaram os alarmes, limitando-os de acordo com a criticidade dos pacientes, e obtiveram, respectivamente, uma redução de $43 \%$ e $44 \%$ do total de alarmes, principalmente os falsos alarmes, minimizando o fenômeno da fadiga de alarmes ${ }^{(5,17)}$.

Podemos inferir que o aumento do número de sinais sonoros de alarmes consistentes disparados na fase pós-parametrização, sofreu forte influência do ajuste adequado dos alarmes das variáveis nas situações em que a parametrização se encontrava com valores incompatíveis com a vida, sem considerar as condições clínicas dos pacientes para o disparo de alarmes e alerta da equipe. Para motivar o aumento no atendimento dos alarmes consistentes, sugere-se como estratégia intervenções multidimensionais nas instituições e o adequado treinamento para melhorar a usabilidade e a segurança desses sistemas de alarmes ${ }^{(18)}$.

Sobre a quantidade de alarmes sonoros atendidos observamos insignificante queda no valor total entre as fases pré e pós-parametrização, onde na fase pré foram 16 e na fase pós 15, entretanto, após a parametrização a maior porcentagem de alarmes sonoros atendidos foi a de alarmes consistentes (60\%), isto é, os
Podemos inferir

que o aumento

do número de

sinais sonoros de

alarmes consistentes

disparados na fase

pós-parametrização,

sofreu forte

influência do ajuste

adequado dos

alarmes das variáveis

nas situações em que

a parametrização

se encontrava

com valores

incompatíveis com a

vida, sem considerar

as condições clínicas

dos pacientes para $o$

disparo de alarmes e

alerta da equipe. alarmes atendidos realmente indicavam necessidade de intervenção.

Após a etapa da intervenção de ajuste dos alarmes observou-se uma queda nos valores de alarmes fatigados no setor, de 124 (25\%) para 90 (21\%), trazendo mais uma evidência sugestiva, que a parametrização pode influenciar na minimização da fadiga de alarmes.

Todas as variáveis, com exceção da FR, apresentaram diminuição nas suas taxas após a parametrização, contudo, esta ação depende do conhecimento adquirido pelo profissional que manuseia o EMA em função das necessidades e particularidades de cada paciente, prevenindo agravos decorrentes do seu uso inadequado ${ }^{(19)}$.

$\mathrm{Na}$ busca de resultados satisfatórios na parametrização, a equipe de enfermagem precisa avaliar as condições atuais dos pacientes e definir os intervalos de alarmes individualmente. Para tal, é fundamental a capacitação dos profissionais e sua aderência às boas práticas de monitoração (11). É importante enfatizar que para melhorar a usabilidade dos EMAs, deve haver interação da equipe assistencial com fabricantes, representantes e, principalmente, a engenharia clínica dos hospitais (16). O autor enfatiza ainda que o conhecimento das alterações determinadas pelos alarmes é essencial para o direcionamento das ações e cuidados de enfermagem com segurança e eficiência.

A partir dos valores obtidos pelo cálculo da OR, é possível inferir que o evento alarme fatigado é desfavorável na fase pós-parametrização, visto que a chance de se encontrar um alarme fatigado na fase pré-parametrização é 1.2 vezes maior que na fase pós. Com relação ao evento alarme consistente considera-se favorável na medida em que a chance de se ter um alarme consistente na fase pré-intervenção é 0.5 vezes menor que após a intervenção.

\section{CONCLUSÕES}

A parametrização dos limites dos alarmes sonoros das variáveis fisiológicas dos pacientes internados no cenário em questão contribuiu para um decréscimo de alarmes 


\section{artigo}

Furtado, M.S.; Bridi, A.C.; Louro, T.Q.; Marques, J.M.; Castro, D.F.; Lyra da Silva, R.C.

Parametrização de alarmes clínicos de monitores multiparâmetros em uma unidade de cuidados crítico

disparados, especialmente dos inconsistentes, e, por conseguinte da fadiga de alarmes.

Este tipo de estudo torna-se imprescindível como um primeiro passo ao mapeamento da unidade, quanto ao fenômeno da fadiga de alarmes, assim como da usabilidade de equipamentos pela equipe. Mapeamento este, que subsidiará posterior capacitação da equipe, adoção de protocolo e rotina referentes à parametrização e usabilidade, que irão concorrer no correto raciocínio clínico na tomada de decisão dos profissionais, favorecendo a vigilância do paciente e consequentemente sua segurança.

\section{REFERÊNCIAS}

1. Padilha KG. Enfermagem em UTI: cuidando do paciente crítico. ed. Manole: 2010.

2. Bridi AC. Fatores determinantes do tempo estímulo-resposta da equipe de enfermagem aos alarmes dos monitolres multiparamétricos em terapia intensiva: implicações para a segurança do paciente grave. [Dissertação]. Rio de Janeiro: Universidade Federal do Estado do Rio de Janeiro, Centro de Ciências Biológicas e da Saúde; 2013.

3. Vasconcellos LG. Avaliação da usabilidade situada de bombas de infusão em uma unidade de cuidados intensivos. [Dissertação]. Rio de Janeiro: Universidade Federal do Estado do Rio de Janeiro, Centro de Ciências Biológicas e da Saúde; 2015.

4. ECRI INSTITUTE: Emergency Care Research Institute. Top 10 health technology hazards for 2019. Pensilvânia (EUA): ECRI INSTITUTE; 2018.

5. Graham KC, Cvach M. Monitor alarm fatigue: standardizing use of physiological monitors and decreasing nuisance alarms. Am J Crit Care [Internet]. 2010 [cited 2019 May 22];19(1):28-34. Available from: https://doi.org/10.4037/ajcc2010651

6. Blum JM, Trember KK. Alarms in the intensive care unit: Too much of a good thing is dangerous: Is it time to add some intelligence to alarms? Critical Care Medicine. 2010; 3(2): 702-703. DOI: 10.1097/CCM.0b013e3181bfe97f

7. Cvach M. Monitor alarm fatigue: an integrative review. Biomed. Instrum Technol [Internet]. 2012 [cited 2019 Mar 20]; 46(4): 268-77. Available from: https://doi.org/110.2345/08998205-46.4.268

8. Ok MC, Hwasoon K, Young WL, Insook C. Clinical alarms in intensive care units: Perceived obstacles of alarm management and alarm fatigue in nurses. Healthc Inform Res. 2016. 22(1): 46-53. DOI: 10.4258/hir.2016.22.1.46

9. Bridi AC, Silva RCL, Farias CCP, Franco AS, Santos VLQ. Tempo estímulo-resposta da equipe de saúde aos alarmes de monitorização na terapia intensiva: implicações para a segurança do paciente grave. Rev Bras Ter Intensiva [Internet]. 2014 [cited 2019 Mar 17]; 26(1): 28-35. Available from: http://www.scielo.br/pdf/ rbti/v26n1/0103-507X-rbti-26-01-0028.pdf

10. Oliveira AEC, Machado AB, Santos ED, Almeida EB. Alarm fatigue and the implications for patient safety. Rev Bras Enferm. 2018. 71(6): 3035-40. DOI: 10.1590/0034-7167-2017-0481

11. Cvach M, Kitchens M, Smith K, Harris P, Flack MN. Customizing Alarm Limits Based on Specific Needs of Patients. Biomedical Instrumentation \& Technology. 2017. 51(3): 227-234. DOI: 10.2345/0899-8205-51.3.227
12. Weisfeldt ML, Becker LB. Ressuscitation after cardiac arrest: a 3 - Phase TimeSensitive Model. JAMA. 2002. 288(23): 3035 3038. DOI: $10.1001 /$ jama.288.23.3035

13. AAMI: Advancing Safety in Medical Technology. Call Alarms. A Siren call for action: priority issues from the medical device alarms SUMMIT [Internet]. Arlington (VA): AAMI; 2011. [cited 2019 May 17]. 48 p. Available from: http://kami.camp9.org/Resources/Pictures/2011\%20Alarms\%20Summit\%20Report.pdf

14. Christensen M, Dodds A, Sauer J, Watts N. Alarm setting for the critically ill patient: a descriptive pilot survey of nurses perceptions of current practice in an Australian Regional Critical Care Unit. Intensive Crit Care Nurs. 2014. 30(4): 204-10. DOI: 10.1016/j.iccn.2014.02.003

15. Farias CCP. Parametrização de alarmes de monitores multiparamétricos em unidade de cuidados intensivos traumato-ortopédicos: contribuições para minimização da fadiga de alarmes. [Dissertação]. Rio de Janeiro: Universidade Federal do Estado do Rio de Janeiro, Centro de Ciências Biológicas e da Saúde; 2015.120p.

16. Costa GL, Lacerda ABM, Marques J. Ruído no contexto hospitalar: impacto na saúde dos profissionais de enfermagem. Rev. CEFAC [Internet]. 2013 [cited 2019 Jan 14]; 15(3): 642-652. Available from: http://www.scielo.br/pdf/rcefac/2013nahe$\mathrm{ad} / 163-11 . \mathrm{pdf}$

17. Assis AP, Oliveira FT, Camerini FG, Silva RCL, Moraes CM. Individualized parameterization of multiparametric monitors alarms in infarcted patients. Rev Bras Enferm. 2019; 72(3): 609-16. doi: http://dx.doi .org/10.1590/0034-7167-2018-0485

18. Sowan AK, Gomez TM, Tarriela AF, Reed CC, Paper BM. Changes in default alarm settings and standard in-service are insufficient to improve alarm fatigue in an intensive care unit: a pilot project. JMIR Hum Factors [Internet]. 2016 [cited 2019 Jan 01]; 3(1):e1. Available from: https:// doi.org/10.2196/humanfactors.509

19. Silva AM, Silva HCA. Segurança do paciente crítico frente à ocorrência de alarmes ventilatórios: condutas do enfermeiro [Trabalho de Conclusão de Curso]. Minas Gerais: Universidade Tiradentes; 2016. 38p.

20. Marta C, Seabra-Junior H, Costa D, Martins G, Silva R, Pereira $\mathrm{L}$. The nursing team before alarm triggering in the neonatal intensive care unit. Rev. pesqui. cuid. fundam. (Online) [Internet]. 2016 [cited 2019 Aug 9]; 8(3): 4773-4779. Available from: http:// www.seer.unirio.br/index.php/cuidadofundamental/article/ view/4054 